\title{
Frequent loss of BTG1 activity and impaired interactions with the Caf1 subunit of the Ccr4-Not deadenylase in non-Hodgkin lymphoma
}

\author{
Hibah Almasmoum $^{\mathrm{a}, \mathrm{b}, \mathrm{c}}$, Blessing Airhihen ${ }^{\mathrm{a}}$, Claire Seedhouse ${ }^{\mathrm{b}}$, and Gerlof Sebastiaan \\ Winkler ${ }^{\mathrm{a}, \mathrm{d}}$
}

a School of Pharmacy, The University of Nottingham, East Drive, University Park, Nottingham NG7 2RD, U.K.

${ }^{\mathrm{b}}$ Department of Haematology, Division of Cancer and Stem Cells, School of Medicine, University of Nottingham Biodiscovery Institute, University Park, Nottingham NG7 2RD, U.K.

${ }^{c}$ Present address: Department of Laboratory Medicine, Faculty of Applied Medical Sciences, Umm Al-Qura University, Makkah, Saudi Arabia. Email: hamasmoum@uqu.edu.sa

d Author for correspondence:

Tel: +44-115-8468457

Email: sebastiaan.winkler@nottingham.ac.uk

Running title: BTG1 mutations in non-Hodgkin lymphoma

Key words: Lymphoma and Hodgkin disease; Ccr4-Not; BTG1; BTG2; mRNA decay

Word count: 3432 words (including figure legends) 


\begin{abstract}
Mutations in the highly similar genes B-cell Translocation Gene 1 (BTG1) and BTG2 were identified in approximately $10-15 \%$ of non-Hodgkin lymphoma cases, which may suggest a direct involvement of $B T G 1$ and $B T G 2$ in malignant transformation. However, it is unclear whether or how disease-associated mutations impair the function of these genes. Therefore, we selected sixteen BTG1 variants based on in silico analysis. We then evaluated (i) the ability of these variants to interact with the known protein-binding partners CNOT7 and CNOT8, which encode the Caf1 catalytic subunit of the Ccr4-Not deadenylase complex; (ii) the activity of the variant proteins in cell cycle progression, (iii) translational repression and (iv) mRNA degradation. Based on these analyses, we conclude that mutations in BTG1 may contribute to malignant transformation and tumour cell proliferation by interfering with its anti-proliferative activity and ability to interact with CNOT7 and CNOT8.
\end{abstract}




\section{Introduction}

The BTG1 and BTG2 proteins are highly similar and mainly divergent in their C-terminus, which is extended in BTG1. Both proteins are part of the human B-cell translocation gene/transducer of ERBB2 (BTG/TOB) family of proteins, which also include BTG3, BTG4, TOB1 and TOB2 [1,2]. A common feature of the BTG/TOB proteins is their ability to inhibit cell cycle progression at the $\mathrm{G}_{1} / \mathrm{S}$ phase and reduce cell proliferation in a variety of cell types [3-7]. The BTG/TOB family members share sequence homology in the BTG domain that comprises the 104-106 N-terminal amino acids [8-10]. The best-characterised role of the BTG domain is in mediating protein-protein interactions $[1,4,11-13,14,15]$. All six BTG/TOB proteins can interact with the highly similar proteins CNOT7 and CNOT8 [4,11-13,16-19], which encode the Caf1 catalytic subunit of the Ccr4-Not deadenylase complex. This complex is involved in the shortening and removal of the mRNA poly(A) tail, which is the initial step in regulated mRNA degradation [20]. Several members of the BTG/TOB family also bind cytoplasmic poly(A)-binding protein PABPC1, which stimulates deadenylation by Caf1 $[17,21,22]$. While TOB1 and TOB2 use a conserved PAM2 motif to interact with the Cterminal domain of PABPC1 [22,23], BTG1 and BTG2 interact with the first RNA-recognition motif of PABPC1 [21]. In addition to the Caf1 paralogues and PABPC1, which are involved in post-transcriptional gene regulation, BTG1 and BTG2 have also been reported to interact with transcription factors, including HoxB9 [24] and nuclear receptors, including ER $\alpha$ [12], and the methyltransferase PRMT1 [25].

Alterations in expression of the $B T G 1$ and $B T G 2$ paralogues are frequently observed in cancer [26-30]. In haematological malignancies, a high frequency of BTG1 deletions occur in cases of acute lymphoblastic leukaemia (ALL) [31]. Moreover, somatic variants of BTG1 and -to a lesser extend- $B T G 2$ have been identified with relatively high frequency in non-Hodgkin's lymphoma [32-37]. However, the effect of the amino acid substitutions on the activity of BTG1 or BTG2 is not known.

Here, we report the effect of lymphoma-associated mutations on the activity of BTG1. First, we used a combination of sequence analysis and molecular modelling to predict the functional consequences of previously identified mutations and selected a sub-set of BTG1 variants for functional analysis. Then, we analysed the ability of BTG1 variants to interact with the Caf 1 (CNOT7/CNOT8) catalytic subunit of the Ccr4-Not complex and functionally assessed the roles of the BTG1 variants in cell cycle progression, translational repression and mRNA degradation.

\section{Materials and methods \\ In silico analysis of BTG1 mutations}

Sequence information about BTG1 variants was collected from the COSMIC database (v65) [38] and additional reports [32-34,36]. The Sorting Intolerant From Tolerant (SIFT) webserver was used to predict the probability of amino acid substitutions as damaging ( $\mathrm{p} 0.05)$ [39].

To obtain a BTG1 structural model, the protein homology/analogy recognition engine (PHYRE) webserver was employed [40], which used the structure of the BTG2 protein as a template (72\% identity; 94\% similarity across 120 amino acids in structure 3E9V) [41]. The BTG1-CNOT7 structural model was prepared using UCSF Chimera [42] by superimposition of the BTG1 model with the structure of the BTG domain of TOB1 in complex with CNOT7 $(\mathrm{PDB} 2 \mathrm{D} 5 \mathrm{R})(\mathrm{rmsd}=1.2 \AA)$ [43].

\section{Plasmid DNA preparation}

Plasmids used in this study are described in the Supplementary Information. 


\section{Yeast two hybrids analysis}

Plasmids pGal4-BD-HA-BTG1 and pGal4-AD-CNOT7 or pGal4-AD-CNOT8 were transformed into yeast strain YRG2 using the LiAc method as described by the manufacturer (Agilent), and grown in selective synthetic drop-out media without leucine or tryptophan (Sigma Aldrich). $\beta$-galactosidase activity of transformants was determined (Beta-Glo Reagent; Promega) and normalised by measuring the optical density (at $600 \mathrm{~nm}$ ) of the yeast cultures [16].

\section{Proliferation assay}

HEK293 cells $\left(n=2 \times 10^{5}\right)$ were seeded onto coverslips placed in 6-well plates and transfected with $1.0 \mu \mathrm{g}$ pCMV5-HA-BTG1 plasmids or empty vector control using the calcium phosphate precipitation method. Forty-eight hours after transfection, cells were pulse labelled for $2 \mathrm{~h}$ using complete medium containing $20 \mu \mathrm{M}$ of the thymidine analogue 5-ethynyl-2'deoxyuridine (EdU), fixed and stained following the manufacturer's protocol (ThermoFisher Scientific; Click-iT EdU Alexa Fluor 594 Imaging Kit C10339). Cell nuclei were stained using Hoechst $33342(5 \mu \mathrm{g} / \mathrm{ml})$. For each slide, at least three images were acquired, each containing 50-150 cells, using an EVOS FL Color Imaging System (Thermo Fisher Scientific). To count S-phase nuclei and the total number of nuclei in an unbiased manner, the ImageJ package (https://imagej.nih.gov/ij/) was used. The number of cells in S-phase determined using this method was comparable to the number of cells in S-phase reported using the EdU labelling method in combination with bivariate flow cytometry [44].

\section{Western blotting}

Cells were harvested in PBS 48 hours post transfection and resuspended in lysis buffer $(50 \mathrm{mM}$ Tris- $\mathrm{HCl} \mathrm{pH} \mathrm{8.0,} 150 \mathrm{mM} \mathrm{NaCl}, 5 \mathrm{mM} \mathrm{MgCl} 2,0.5 \mathrm{mM}$ EDTA, 5\% glycerol, $1 \% \mathrm{NP}-40,1 \mathrm{mM}$ dithiotreitol). Soluble proteins were separated by $14 \%$ SDS-PAGE and expression of HABTG1 variants was confirmed by western blotting using rat monoclonal antibody 3F10 (HA epitope tag, Roche, diluted 1:500). Goat polyclonal antibody C-20 (anti- $\gamma$ tubulin, Santa Cruz sc-7396, diluted 1:1000) was used as a loading control.

\section{RNA tethering assay}

HEK293 cells $(n=80,000)$ were seeded into 12-well culture plates and transfected using the calcium phosphate method with $0.5 \mu \mathrm{g}$ of plasmid pCI $\lambda \mathrm{N}-\mathrm{BTG} 1$ (or empty vector) and $0.5 \mu \mathrm{g}$ of the reporter plasmid pRL-5BoxB. Cells were harvested 24 hours post-transfection before measuring luciferase activity using the BioLux Gaussia Luciferase Assay Kit (New England Biolabs, E3300S). Luciferase activity was normalised to total protein content determined using a Bradford assay (Sigma).

Reverse transcriptase-quantitative PCR was used to measure the level of reporter mRNA. The levels of luciferase mRNA were determined using a TaqMan assay and GAPDH as a control. Further details are available in the Supplementary Information.

\section{Results}

\section{In silico evaluation of somatic mutations in BTG1}

Details of lymphoma-associated mutations in $B T G 1$ were collected from literature reports and the Catalogue of Somatic Mutations In Cancer database (COSMIC) [38]. After discarding synonymous mutations, twenty-five BTG1 variants were identified in non-Hodgkin lymphoma (NHL) [32-36]. The majority of BTG1 variants are clustered in the BTG domain (19/25). 
To evaluate the potential effect of amino acid substitutions on protein function, we used the Sorting Intolerant From Tolerant (SIFT) algorithm as a first step [39]. This algorithm is based on evolutionary conservation of protein sequences and predicts the potential impact of amino acid substitutions on protein function by calculating the probability for the altered amino acid to occur at that position. Overall, $60 \%$ of BTG1 variants (15/25) were predicted to be damaging using SIFT ( $\mathrm{p}$ < 0.05) (Figure 1A; Table 1).

\section{Structural analysis of BTG1 variants}

To further understand the possible effect of the disease-associated amino acid substitutions, a structural model of the BTG1-Caf1/CNOT7 complex was prepared. First, a BTG1 homology model (residues 11-129) was prepared based on the structure of BTG2 [40]. Then, the BTG1 model was superimposed on the BTG domain of TOB1 of the TOB1-Caf1/CNOT7 structure (Figure 1B) [43]. Mapping of the lymphoma-associated variants on the structural model indicated that most tolerated amino acid substitutions were located in the $\alpha$-helical $\mathrm{N}$-terminal region away from the interaction surface with Caf1/CNOT7. By contrast, damaging variants were also present in the $\beta$-stranded region that forms the interface with Caf1/CNOT7 (Figure 1B). Similar results were obtained when BTG2 mutations were analysed (Supplementary Figure S1).

\section{Lymphoma-associated $B T G 1$ amino acid substitutions affect the interaction with human Caf1}

To assess the possible effects of the amino acid substitutions on the function of BTG1, we selected 16 single amino acid variants based on the in silico analysis and investigated whether these variants were able to interact with the Caf1/CNOT7 and Caf1/CNOT8 subunits of the Ccr4-Not complex. To this end, we used the yeast two-hybrid assay based on the Gal4 DNAbinding domain (BD) fused to BTG1 and the Gal4 activation domain (AD) fused to CNOT7 or CNOT8 (Figure 2). Robust $\beta$-galactosidase expression ( $>20$-fold induction compared to background) was observed when Gal4-BD-HA-BTG1 was co-expressed with Gal4-ADCNOT7 or Gal4-AD-CNOT8 (Figure 2).

Eight amino acid substitutions, R27H and F40C, which contain amino acid alterations in $\alpha-$ helices $\alpha 1$ and $\alpha 2$ (Figure 2A and 2D), L104H and I115V, located in $\beta$-sheets $\beta 2$ and $\beta 3$ (Figure 2B and 2E), M11I (loop L1), P58L (loop L1), G66V (helix $\alpha 3$ ) and N73K (loop L4) (Figure 2C and $\mathbf{2 F}$ ), resulted in very low $\beta$-galactosidase activity and were therefore strongly impaired the interaction with CNOT7 and CNOT8.

Expression of BTG1 variants Q36H ( $\alpha 2)$ and L37M ( $\alpha 2)$ did not result in reduced $\beta$ galactosidase activity when co-expressed with AD-CNOT7 (Figure 2A), although reduced reporter activity was observed when expressed with AD-CNOT8 (Figure 2D). Variants $\Delta$ N10 BTG1, H2Y and E117D (loop L8) were able to stimulate $\beta$-galactosidase activity similarly to wild type BTG1, although BTG1 E117D was marginally disrupted in the interaction with CNOT8. Interestingly, E59D (loop L2), which was selected due to the fact that the conservative amino acid change was located close to the surface of the interaction interface, showed a partial disruption in the interaction with CNOT7 and CNOT8 (Figure 2C and 2F). Taken together, these results indicate that the majority of the BTG1 variants displayed a reduced ability to interact with the CNOT7 and CNOT8 subunits of the Ccr4-Not complex. Similar results were obtained when BTG2 mutations were analysed (Supplementary Figure S2).

The effect of lymphoma-derived BTG1 mutations on the regulation of cell proliferation Next, we investigated whether the ability of BTG1 to inhibit cell cycle progression was affected by lymphoma-associated amino acid substitutions. To this end, HEK293T cells were 
transfected with HA-BTG1 expression vectors and the number of cells in S-phase was determined via incorporation of the thymidine analogue EdU (Figure 3).

Compared to control-transfected cells, cells expressing wild type BTG1 consistently showed a reduction of cells in S-phase as expected based on its anti-proliferative activity (Figure 3A). By contrast, expression of variants R27H, F25C, F40C (Figure 3A), L104H, I115V (Figure 3B), M11I, P58L, G66V (Figure 3C), N73K and E117D (Figure 3D), L37M and L94V (Figure 3F) did not inhibit cell cycle progression. In each case, western blot analysis confirmed expression levels similar to wild type BTG1, except for variant F40C, which consistently showed reduced expression, indicating that this variant may be unstable.

Expression of variants $\mathrm{H} 2 \mathrm{Y}$ and $\mathrm{Q} 36 \mathrm{H}$ resulted in reduced cell cycle progression to a level similar to that of wild type BTG1 (Figure 3E). This was also observed with the BTG1 variant lacking the first methionine start codon, which is predicted to result in the deletion of the first ten amino acids (BTG1 $\Delta$ N10) (Figure 3F). Variant E59D, which was predicted to be tolerated by the SIFT algorithm, has reduced affinity for CNOT7 and CNOT8, and is also unable to inhibit cell cycle progression (Figure 3F).

Taken together, these results indicate that lymphoma-associated amino acid substitutions frequently interfere with the ability of BTG1 to inhibit cell cycle progression.

\section{Regulation of mRNA translation and degradation of a tethered reporter mRNA by BTG1 variants}

Because of the high similarity between BTG1 and BTG2, it is expected that the antiproliferative function of these proteins is mediated through their role in mRNA decay and translational repression [16,21]. Therefore, we tested the activity of the selected BTG1 variants in mRNA degradation and translational repression. Thus, we expressed BTG1 fused to the $\lambda N$ peptide together with a luciferase reporter mRNA containing five Box B sequence motifs located in the 3' untranslated region. As expected, expression of $\lambda \mathrm{N}-\mathrm{HA}-\mathrm{BTG} 1$ repressed translation of a tethered reporter mRNA as measured by luciferase activity (Figure 4A-F). The decrease in luciferase activity was due to degradation of the tethered reporter, as the mRNA levels of the reporter were also reduced by a similar amount (Figure 4A-F).

Next, the sixteen BTG1 variants were expressed as $\lambda \mathrm{N}$-fusion proteins, and their ability to repress translation and induce degradation of a tethered luciferase reporter evaluated. Variants F40C (Figure 4A) and G66V (Figure 4C) showed impaired ability to repress luciferase activity and degrade the reporter mRNA. In case of F40C, residual activity remained, whereas G66V was unable to inhibit translation and induce degradation of the reporter mRNA.

The activities of variants H2Y, Q36H (Figure 4E) and $\Delta$ N10 (Figure 4F) were comparable to that of wild type $\lambda \mathrm{N}-\mathrm{HA}-\mathrm{BTG} 1$ and both luciferase activity and reporter mRNA levels were reduced compared to empty vector-transfected cells. Upon expression of $\lambda \mathrm{N}-\mathrm{HA}-\mathrm{BTG} 1$ L104H, a modest reduction in luciferase activity was observed compared to wild type BTG1, while the reduction of reporter mRNA levels compared to empty vector control was moderate (Figure 4B).

Surprisingly, expression of $\lambda$ N-HA-BTG1 containing amino acid substitutions F25C, R27H (Figure 4A), I115V (Figure 4B), M11I, P58L (Figure 4C), N73K, E117D, E59D (Figure 4D), L37M, and L94V (Figure 4F) resulted in full repression of luciferase activity and degradation of the reporter mRNA even though these variants were impaired in their interactions with CNOT7 and/or CNOT8 (Figure 2), and unable to inhibit cell proliferation (Figure 3). For all variants, western blot analysis indicated that they were all expressed to similar levels as $\lambda \mathrm{N}$-HA-BTG1. Comparable results were obtained when $B T G 2$ mutations were analysed (Supplementary Figure S3). 


\section{Pleiotropic effects of amino acid substitutions on BTG1 activity}

Because the analysed mutations displayed pleiotropic effects on BTG1 activities, we used hierarchical clustering to sort the selected BTG1 variants and identified four groups (Figure 5). First, three mutations (resulting in amino acid substitutions H2Y, Q36K and a deletion of the ten N-terminal amino acids) did not interfere with BTG1 activities under the conditions used (group I). BTG1 variants in the second group (L37M, L94V, E117D, F25C and E59D), retained a moderate ability to bind the Caf1 paralogues CNOT7 and CNOT8, and displayed substantial defects in the ability of BTG1 to reduce cell cycle progression (group II). In group III (I115V, R27H, M11I, and N73K), variants also displayed defects in the ability of BTG1 to reduce cell cycle progression, and displayed substantial defects in the interaction with CNOT7 and CNOT8. Finally, three mutations (resulting in amino acid substitutions G66V, L104H and F40C) severely interfered with BTG1 activity and behaved under the tested conditions as the controls lacking BTG1 expression (group IV). In addition to defects in Caf1 (CNOT7/CNOT8) binding and impaired ability to inhibit cell cycle progression, variants in this group were also unable to induce mRNA degradation and inhibit mRNA translation when tethered to a reporter mRNA (Figure 5).

\section{Discussion}

In recent years, several reports revealed frequent mutations in BTG1 and -less commonlythe highly similar gene BTG2 in non-Hodgkin lymphoma [32-36]. Here, we show that lymphoma-associated point mutations in BTG1 frequently affect the function of the gene product.

The observation that BTG1 variants with impaired binding to the Caf1 paralogues CNOT7 and CNOT8 are also defective in the ability to inhibit cell cycle progression correlates well with previous observations of BTG2, which used designed mutations that disrupt the interaction with Caf1 [16,21]. Contrary to these studies, however, some BTG1 variants are still able to induce degradation of a tethered reporter even though they are unable to interact with Caf1. In this respect, it should be noted that combined knockdown of CNOT7 and CNOT8 did not fully rescue mRNA levels and luciferase activity of the reporter when tethered by BTG2 [16]. It has been reported that BTG1 and BTG2 also interact with other proteins, including HoxB9, PRMT1 and nuclear receptors, such as ER $\alpha$, that are involved in cancer $[12,24,25]$. Inactivating mutations in BTG1 and BTG2 may also impact on proteinprotein interactions with these partners and contribute to the tumour phenotype.

Due to their similarity, it is likely that a significant proportion of mutations in BTG2 would interfere with the activity of the gene product in a similar manner as BTG1 mutations. Reduced expression of BTG1 or BTG2 is observed in several tumour types [26-30], and downregulation of BTG1 in lymphoma is a common feature in c-MYC induced lymphoma [45]. The mIR-17-92 cluster, which is frequently amplified in B-cell lymphomas [46], is upregulated by increased c-MYC activity. In turn, this cluster suppresses the expression of several genes, including BTG1, that contribute to the tumour phenotype. In agreement with an important role of BTG1 in lymphoma, knockdown of BTG1 can overcome the proliferative arrest following inactivation of c-MYC [45]. It may therefore be that inactivation of $B T G 1$ or $B T G 2$ by single base pair mutations contributes to tumour development of non-Hodgkin lymphoma. It may be that both proteins can substitute each other's function and it could be speculated that inactivation of BTG1 or BTG2 by single base mutations reduces their combined activity that is functionally similar to reduced expression of $B T G 1$ or $B T G 2$ observed in other tumour types. In agreement with this notion, there is a 
statistically significant overlap between inactivation of BTG1 and BTG2 in diffuse large B cell lymphoma [37].

Altered expression of other members of the BTG/Tob family of proteins are also implicated in cancer [47]. Altered expression of BTG3 and changes in the phosphorylation status of TOB 1 are frequently observed in lung cancer $[48,49]$. On the other hand, high expression of TOB1 is associated with poor prognosis in breast cancer [50,51]. Thus, further understanding of the molecular mechanism(s) by which these proteins exert their effects will lead to an improved model for the role of these proteins in cancer development and progression.

\section{Acknowledgements}

The Saudi Arabia Ministry of Higher Education and the Umm Al-Qura University (Makkah, Saudi Arabia) are acknowledged for their generous funding of the scholarship of H.A. B.A. was a recipient of a Faculty for the Future scholarship of the Schlumberger Foundation. Dr Laura Corbo (Lyon, France) is gratefully acknowledged for contributing the pSG5-FlagBTG1 expression plasmid.

\section{Disclosure of interest}

The authors report no conflict of interest 


\section{References}

1. Winkler GS. The mammalian anti - proliferative BTG/Tob protein family. Journal of cellular physiology 2010;222:66-72.

2. Yuniati L, Scheijen B, van der Meer LT, van Leeuwen FN. Tumor suppressors BTG1 and BTG2: Beyond growth control. J. Cell. Physiol. 2018;234:5379-5389.

3. Buanne P, Corrente G, Micheli L, et al. . Cloning of PC3B, a novel member of the PC3/BTG/TOB family of growth inhibitory genes, highly expressed in the olfactory epithelium. Genomics 2000;68:253-263.

4. Ikematsu N, Yoshida Y, Kawamura-Tsuzuku J, et al. . Tob2, a novel anti-proliferative Tob/BTG1 family member, associates with a component of the CCR4 transcriptional regulatory complex capable of binding cyclin-dependent kinases. Oncogene 1999;18.

5. Matsuda S, Kawamura-Tsuzuku J, Ohsugi M, et al. . Tob, a novel protein that interacts with p185erbB2, is associated with anti-proliferative activity. Oncogene 1996;12:705-713.

6. Montagnoli A, Guardavaccaro D, Starace G, Tirone F. Overexpression of the nerve growth factor-inducible PC3 immediate early gene is associated with growth inhibition. Cell growth \& differentiation: the molecular biology journal of the American Association for Cancer Research 1996;7:1327-1336.

7. Rouault J, Rimokh R, Tessa C, et al. . BTG1, a member of a new family of antiproliferative genes. The EMBO journal 1992;11:1663.

8. Guardavaccaro D, Corrente G, Covone F, et al. . Arrest of G1-S progression by the p53-inducible gene PC3 is Rb dependent and relies on the inhibition of cyclin D1 transcription. Molecular and cellular biology 2000;20:1797-1815.

9. Rouault J-P, Falette N, Guéhenneux F, et al. . Identification of BTG2, an antiproliferative p53-dependent component of the DNA damage cellular response pathway. Nature Genetics 1996;14:482-486.

10. Yang X, Morita M, Wang H, et al. . Crystal structures of human BTG2 and mouse TIS21 involved in suppression of CAF1 deadenylase activity. Nucleic acids research 2008;36:6872-6881.

11. Rouault J-P, Prévôt D, Berthet C, et al. . Interaction of BTG1 and p53-regulated BTG2 Gene Products with mCaf1, the Murine Homolog of a Component of the Yeast CCR4 Transcriptional Regulatory Complex. Journal of Biological Chemistry 1998;273:22563-22569.

12. Prévôt D, Morel A-P, Voeltzel T, et al. . Relationships of the Antiproliferative Proteins BTG1 and BTG2 with CAF1, the Human Homolog of a Component of the Yeast CCR4 Transcriptional Complex INVOLVEMENT IN ESTROGEN RECEPTOR $\alpha$ SIGNALING PATHWAY. Journal of Biological Chemistry 2001;276:9640-9648.

13. Yoshida Y, Hosoda E, Nakamura T, Yamamoto T. Association of ANA, a member of the antiproliferative Tob family proteins, with a Cafl component of the CCR4 transcriptional regulatory complex. Japanese journal of cancer research 2001;92:592596. 
14. Busson M, Carazo A, Seyer P, et al. . Coactivation of nuclear receptors and myogenic factors induces the major BTG1 influence on muscle differentiation. Oncogene 2005;24:1698-1710.

15. Ou YH, Chung PH, Hsu FF, Sun TP, Chang WY, Shieh SY. The candidate tumor suppressor BTG3 is a transcriptional target of p53 that inhibits E2F1. The EMBO journal 2007;26:3968-3980.

16. Doidge R, Mittal S, Aslam A, Winkler GS. The anti-proliferative activity of BTG/TOB proteins is mediated via the Caf1a (CNOT7) and Caf1b (CNOT8) deadenylase subunits of the Ccr4-not complex. PLoS One 2012;7:e51331.

17. Ezzeddine N, Chen C-YA, Shyu A-B. Evidence providing new insights into TOBpromoted deadenylation and supporting a link between TOB's deadenylationenhancing and antiproliferative activities. Molecular and cellular biology 2012;32:1089-1098.

18. Pasternak M, Pfender S, Santhanam B, Shuh M. The BTG4 and CAF1 complex prevents the spontaneous activation of eggs by deadenylating maternal mRNAs. Open Biol. 2016;6:160184.

19. Yu C, Ji SY, Sha QQ, et al. . BTG4 is a meiotic cell cycle-coupled maternal-zygotictransition licensing factor in oocytes. Nat. Struct. Mol. Biol. 2016;23:387-394.

20. Wahle E, Winkler GS. RNA decay machines: deadenylation by the Ccr4-Not and Pan2-Pan3 complexes. Biochimica et Biophysica Acta (BBA)-Gene Regulatory Mechanisms 2013;1829:561-570.

21. Stupfler B, Birck C, Séraphin B, Mauxion F. BTG2 bridges PABPC1 RNA-binding domains and CAF1 deadenylase to control cell proliferation. Nature communications 2016;7:10811.

22. Ezzeddine N, Chang T-C, Zhu W, et al. . Human TOB, an antiproliferative transcription factor, is a poly (A)-binding protein-dependent positive regulator of cytoplasmic mRNA deadenylation. Molecular and cellular biology 2007;27:77917801.

23. Funakoshi Y, Doi Y, Hosoda N, et al. . Mechanism of mRNA deadenylation: evidence for a molecular interplay between translation termination factor eRF3 and mRNA deadenylases. Genes \& development 2007;21:3135-3148.

24. Prévôt D, Voeltzel T, Birot A-M, et al. . The leukemia-associated protein Btg1 and the p53-regulated protein Btg2 interact with the homeoprotein Hoxb9 and enhance its transcriptional activation. Journal of Biological Chemistry 2000;275:147-153.

25. Lin W-J, Gary JD, Yang MC, Clarke S, Herschman HR. The mammalian immediateearly TIS21 protein and the leukemia-associated BTG1 protein interact with a proteinarginine N-methyltransferase. Journal of Biological Chemistry 1996;271:1503415044.

26. Kanda M, Sugimoto H, Nomoto S, et al. . B-cell translocation gene 1 serves as a novel prognostic indicator of hepatocellular carcinoma. J. Oncol. 2015;46:641-648.

27. Kanda M, Oya H, Nomoto S, et al. . Diversity of clinical implication of B-cell translocation gene 1 expression by histopathologic and anatomic subtypes of gastric cancer. Digestive diseases and sciences 2015;60:1256-1264. 
28. Kawakubo H, Brachtel E, Hayashida T, et al. . Loss of B-cell translocation gene-2 in estrogen receptor-positive breast carcinoma is associated with tumor grade and overexpression of cyclin d1 protein. Cancer research 2006;66:7075-7082.

29. Struckmann K, Schraml P, Simon R, et al. . Impaired expression of the cell cycle regulator BTG2 is common in clear cell renal cell carcinoma. Cancer research 2004;64:1632-1638.

30. Ficazzola MA, Fraiman M, Gitlin J, et al. . Antiproliferative B cell translocation gene 2 protein is down-regulated post-transcriptionally as an early event in prostate carcinogenesis. Carcinogenesis 2001;22:1271-1279.

31. Waanders E, Scheijen B, van der Meer LT, et al. . The origin and nature of tightly clustered BTG1 deletions in precursor B-cell acute lymphoblastic leukemia support a model of multiclonal evolution. PLoS Genet 2012;8:e1002533.

32. Morin RD, Mendez-Lago M, Mungall AJ, et al. . Frequent mutation of histonemodifying genes in non-Hodgkin lymphoma. Nature 2011;476:298-303.

33. Lohr JG, Stojanov P, Lawrence MS, et al. . Discovery and prioritization of somatic mutations in diffuse large B-cell lymphoma (DLBCL) by whole-exome sequencing. Proceedings of the National Academy of Sciences 2012;109:3879-3884.

34. Love C, Sun Z, Jima D, et al. . The genetic landscape of mutations in Burkitt lymphoma. Nature genetics 2012;44:1321-1325.

35. Walker BA, Wardell CP, Melchor L, et al. . Intraclonal heterogeneity and distinct molecular mechanisms characterize the development of $t(4 ; 14)$ and $t(11 ; 14)$ myeloma. Blood 2012;120:1077-1086.

36. Zhang J, Grubor V, Love CL, et al. . Genetic heterogeneity of diffuse large B-cell lymphoma. Proceedings of the National Academy of Sciences 2013;110:1398-1403.

37. Reddy A, Zhang J, Davis NS, et al. . Genetic and Functional Drivers of Diffuse Large B Cell Lymphoma. Cell 2017;171:481-494.e415.

38. Forbes SA, Bindal N, Bamford S, et al. . COSMIC: mining complete cancer genomes in the Catalogue of Somatic Mutations in Cancer. Nucleic acids research 2011;39:D945-D950.

39. Kumar P, Henikoff S, Ng PC. Predicting the effects of coding non-synonymous variants on protein function using the SIFT algorithm. Nature protocols 2009;4:10731081.

40. Kelley LA, Mezulis S, Yates CM, Wass MN, Sternberg MJ. The Phyre2 web portal for protein modeling, prediction and analysis. Nature protocols 2015;10:845-858.

41. Sampathkumar P, Romero R, Wasserman S, et al. . Crystal structure of human B-cell Translocation Gene 2 (BTG2). 2008.

42. Meng EC, Pettersen EF, Couch GS, Huang CC, Ferrin TE. Tools for integrated sequence-structure analysis with UCSF Chimera. BMC bioinformatics 2006;7:339.

43. Horiuchi M, Takeuchi K, Noda N, et al. . Structural basis for the antiproliferative activity of the Tob-hCaf1 complex. Journal of Biological Chemistry 2009;284:1324413255 .

44. Clarke ST, Calderon V, Bradford JA. Click chemistry for analysis of cell proliferation in flow cytometry. Current Protocols in Cytometry 2017;82:7.49.41-47.49.30. 
45. Li Y, Choi PS, Casey SC, Dill DL, Felsher DW. MYC through miR-17-92 suppresses specific target genes to maintain survival, autonomous proliferation, and a neoplastic state. Cancer Cell 2014;26:262-272.

46. He L, Thomson JM, Hemann MT, et al. . A microRNA polycistron as a potential human oncogene. Nature 2005;435:828-833.

47. Bai Y, Qiao L, Xie N, Shi Y, Liu N, Wang J. Expression and prognosis analyses of the Tob/BTG antiproliferative (APRO) protein family in human cancers. PLoS One 2007;12:e0184902.

48. Iwanaga K, Sueoka N, Sato A, et al. . Alteration of expression or phosphorylation status of tob, a novel tumor suppressor gene product, is an early event in lung cancer. Cancer letters 2003;202:71-79.

49. Yoneda M, Suzuki T, Nakamura T, et al. . Deficiency of antiproliferative family protein Ana correlates with development of lung adenocarcinoma. Cancer science 2009; 100:225-232.

50. Faraji F, Hu Y, Yang HH, et al. . Post-transcriptional control of tumor cell autonomous metastatic potential by CCR4-NOT deadenylase CNOT7. PLoS Genet 2016;12:e1005820.

51. Helms MW, Kemming D, Contag CH, et al. . TOB1 is regulated by EGF-dependent HER2 and EGFR signaling, is highly phosphorylated, and indicates poor prognosis in node-negative breast cancer. Cancer research 2009;69:5049-5056. 


\section{Tables}

Table 1. List of BTG1 variants evaluated using the SIFT algorithm in different types of lymphoma.

\begin{tabular}{|c|c|c|c|c|c|c|c|c|}
\hline No & Position & $\begin{array}{l}\text { Amino acid } \\
\text { substitution }\end{array}$ & Mutation & Codons & $\mathbf{p}$ & Prediction & $\begin{array}{c}\text { Lymphoma } \\
\text { sub-type }^{\text {a }}\end{array}$ & Ref. \\
\hline 1 & 1 & $\mathrm{M} \rightarrow \mathrm{I}$ & $\mathrm{G}>\mathrm{A}$ & ATG $>$ ATA & 0 & Damaging & DLBCL & 33 \\
\hline 2 & 2 & $\mathrm{H} \rightarrow \mathrm{Y}$ & $\mathrm{C}>\mathrm{T}$ & CAT $>$ TAT & 0.037 & Damaging & $\mathrm{ABC}, \mathrm{GCB}$ & 32 \\
\hline 3 & 3 & $\mathrm{P} \rightarrow \mathrm{R}$ & $\mathrm{C}>\mathrm{G}$ & $\mathrm{CCC}>\mathrm{CGC}$ & 0.069 & Tolerated & DLBCL & 33 \\
\hline 4 & 5 & $\mathrm{Y} \rightarrow \mathrm{H}$ & $\mathrm{T}>\mathrm{C}$ & $\mathrm{TAC}>\mathrm{CAC}$ & 0.409 & Tolerated & DLBCL & 33 \\
\hline 5 & 11 & $\mathrm{M} \rightarrow \mathrm{I}$ & $\mathrm{G}>\mathrm{A}$ & ATG > ATA & 0.028 & Damaging & DLBCL & 33 \\
\hline 6 & 23 & $\mathrm{~S} \rightarrow \mathrm{A}$ & $\mathrm{T}>\mathrm{G}$ & $\mathrm{TCC}>\mathrm{GCC}$ & 0.427 & Tolerated & DLBCL & 33 \\
\hline 7 & 25 & $\mathrm{~F} \rightarrow \mathrm{C}$ & $\mathrm{T}>\mathrm{G}$ & $\mathrm{TTT}>\mathrm{TGT}$ & 0.001 & Damaging & DLBCL & 33 \\
\hline 8 & 27 & $\mathrm{R} \rightarrow \mathrm{H}$ & $\mathrm{G}>\mathrm{A}$ & $\mathrm{CGC}>\mathrm{CAC}$ & 0.039 & Damaging & GCB & 32 \\
\hline 9 & 31 & $\mathrm{~L} \rightarrow \mathrm{F}$ & $\mathrm{C}>\mathrm{T}$ & CTC>TTC & 0.726 & Tolerated & DLBCL & 33 \\
\hline 10 & 36 & $\mathrm{Q} \rightarrow \mathrm{H}$ & $\mathrm{G}>\mathrm{C}$ & $\mathrm{CAG}>\mathrm{CAC}$ & 0.006 & Damaging & ABC & 32 \\
\hline 11 & 37 & $\mathrm{~L} \rightarrow \mathrm{M}$ & $\mathrm{C}>\mathrm{A}$ & $\mathrm{CTG}>\mathrm{ATG}$ & 0.02 & Damaging & GCB & 32,35 \\
\hline 12 & 37 & $\mathrm{~L} \rightarrow \mathrm{L}$ & $\mathrm{C}>\mathrm{T}$ & $\mathrm{CTG}>\mathrm{TTG}$ & 1 & Tolerated & $\begin{array}{l}\text { Lymphoid } \\
\text { neoplasm, } \\
\text { MM }\end{array}$ & 33,35 \\
\hline 13 & 38 & $\mathrm{Q} \rightarrow \mathrm{E}$ & $\mathrm{C}>\mathrm{G}$ & $\mathrm{CAG}>\mathrm{GAG}$ & 0.444 & Tolerated & GCB & 32 \\
\hline 14 & 40 & $\mathrm{~F} \rightarrow \mathrm{C}$ & $\mathrm{T}>\mathrm{G}$ & TTC>TGC & 0 & Damaging & DLBCL & 33 \\
\hline 15 & 46 & $E \rightarrow D$ & $\mathrm{G}>\mathrm{C}$ & $\mathrm{GAG}>\mathrm{GAC}$ & 0.239 & Tolerated & GCB & 32 \\
\hline 16 & 46 & $\mathrm{E} \rightarrow \mathrm{Q}$ & $\mathrm{G}>\mathrm{C}$ & $\mathrm{GAG}>\mathrm{CAG}$ & 0.155 & Tolerated & DLBCL & 33 \\
\hline 17 & 58 & $\mathrm{P} \rightarrow \mathrm{L}$ & $\mathrm{C}>\mathrm{T}$ & $\mathrm{CCA}>\mathrm{CTA}$ & 0 & Damaging & ABC & 32 \\
\hline 18 & 59 & $\mathrm{E} \rightarrow \mathrm{D}$ & $\mathrm{A}>\mathrm{T}$ & $\mathrm{GAA}>\mathrm{GAT}$ & 0.541 & Tolerated & DLBCL & 33 \\
\hline 19 & 66 & $\mathrm{G} \rightarrow \mathrm{V}$ & $\mathrm{G}>\mathrm{T}$ & GGT>GTT & 0.001 & Damaging & Unknown & 36 \\
\hline 20 & 67 & $\mathrm{Y} \rightarrow \mathrm{Y}$ & $\mathrm{C}>\mathrm{T}$ & TAC $>$ TAT & 1 & Tolerated & $\begin{array}{c}\text { Lymphoid } \\
\text { neoplasm, } \\
\text { MM }\end{array}$ & 35 \\
\hline 21 & 73 & $\mathrm{~N} \rightarrow \mathrm{K}$ & $\mathrm{C}>\mathrm{A}$ & $\mathrm{AAC}>\mathrm{AAA}$ & 0.001 & Damaging & Unknown & 36 \\
\hline 22 & 94 & $\mathrm{~L} \rightarrow \mathrm{V}$ & $\mathrm{C}>\mathrm{G}$ & CTG $>$ GTG & 0.002 & Damaging & GCB & 32 \\
\hline 23 & 104 & $\mathrm{~L} \rightarrow \mathrm{H}$ & $\mathrm{T}>\mathrm{A}$ & $\mathrm{CTC}>\mathrm{CAC}$ & 0 & Damaging & DLBCL & 36 \\
\hline 24 & 115 & $\mathrm{I} \rightarrow \mathrm{V}$ & $A>G$ & $\mathrm{ATT}>\mathrm{GTT}$ & 0.003 & Damaging & DLBCL & 33 \\
\hline 25 & 117 & $\mathrm{E} \rightarrow \mathrm{D}$ & $\mathrm{G}>\mathrm{T}$ & $\mathrm{GAG}>\mathrm{GAT}$ & 0.022 & Damaging & DLBCL & 33 \\
\hline 26 & 149 & $\mathrm{C} \rightarrow *$ & $\mathrm{~T}>\mathrm{A}$ & TGT> TGA & - & - & DLBCL & 36 \\
\hline 27 & 165 & $\mathrm{~N} \rightarrow \mathrm{S}$ & $\mathrm{A}>\mathrm{G}$ & $\mathrm{AAT}>\mathrm{AGT}$ & 0.46 & Tolerated & DLBCL & 33 \\
\hline
\end{tabular}

${ }^{a}$ Follicular Follicular lymphoma (FL), diffuse large B-cell lymphoma (DLBCL), germinal centre B-cell (GCB), activated B-cell (ABC) and multiple myeloma (MM). SIFT prediction software $v 1.03$ was used [39]. The zygosity was not identified in all BTG2 mutations. 


\section{Figure legends}

Figure 1. In silico analysis of lymphoma-associated BTG1 variants.

(A) Overview of lymphoma-associated mutations in BTG1. Mutations predicted to be damaging by the SIFT algorithm [39] are shown in red $(\mathrm{p}<0.05)$; mutations in blue are predicted to be tolerated. The conserved BTG domain is shown in light grey; conserved regions Box A, B and C are shown in dark grey. (B) Structural mapping of BTG1 variants. The BTG1 model was generated by homology modelling [40]. The BTG1 model (light khaki) was then superimposed on the BTG domain of TOB1 in complex with CNOT7 (white) [43].

Figure 2. Impaired interactions between lymphoma-associated BTG1 variants and the CNOT7 and CNOT8 deadenylase subunits of the Ccr4-Not complex.

(A-C) Yeast two-hybrid interactions between CNOT7 and BTG1 variants located in (A) $\alpha$ helical regions, (B) $\beta$-sheets, or (C) loops. (D-F) Yeast two-hybrid interactions between CNOT8 and BTG1 variants located in (D) $\alpha$-helical regions, (E) $\beta$-sheets, or (F) loops. Error bars indicate the standard error of the mean $(n=3)$. P values were calculated using a one-way Anova and Dunnett's post-hoc test. $* \mathrm{p}<0.05, * * \mathrm{p}<0.01, * * * \mathrm{p}<0.001, * * * * \mathrm{p}<0.0001$.

Figure 3. Lack of anti-proliferative activity in lymphoma-associated BTG1 variants.

(A-F) Effect of expression of lymphoma-associated BTG1 variants on cell cycle progression. Top panels, lysates of transfected cells were separated by SDS-PAGE and immunoblots probed with anti-HA antibodies. Antibodies recognising $\gamma$-tubulin were used as loading controls. Bottom panels, the percentage of cells in S-phase was determined by incorporation of the thymidine analogue EdU in HEK293T cells expressing BTG1 variants. Error bars indicate the standard error of the mean $(n=3)$. $\mathrm{P}$ values were calculated using a one-way Anova and Tukey's post-hoc test. $* \mathrm{p}<0.05$, ** $\mathrm{p}<0.01$, *** $\mathrm{p}<0.001$ and $* * * * \mathrm{p}<0.0001$ compared to control cells transfected with pCMV5-HA-BTG1.

\section{Figure 4. Lymphoma-associated amino acid substitutions of BTG1 do not generally} interfere with degradation of a tethered reporter mRNA.

(A-F) Top panels, lysates of transfected cells were separated by SDS-PAGE and immunoblots probed with anti-HA antibodies to detect the $\lambda \mathrm{N}-\mathrm{HA}-\mathrm{BTG} 1$ variants. Antibodies recognising $\gamma$-tubulin were used as loading controls. Bottom panels, luciferase activity and reporter mRNA levels obtained after expression of wild type BTG1 and lymphoma-associated BTG1 variants. HEK293T cells were co-transfected with a reporter plasmid containing a Renilla luciferase expression cassette containing five box B sequences in the $3^{\prime}$ UTR and a plasmid expressing $\lambda \mathrm{N}-\mathrm{HA}-\mathrm{BTG} 1$ fusion proteins. Levels of the luciferase reporter mRNA were determined by reverse transcriptase-quantitative PCR. Error bars indicate the standard error of the mean $(n=3)$. P values were calculated by a one-way ANOVA with Tukey's post-hoc test. $* \mathrm{P}<0.05$, *** $\mathrm{p}<0.001$ and $* * * * \mathrm{p}<0.0001$ (compared to the cells expressing wild type $\lambda \mathrm{N}-\mathrm{HA}-\mathrm{BTG} 1$ ).

\section{Figure 5. Pleiotropic effects of amino acid substitutions on BTG1 activity.}

Heat map representing amino acid substitutions clustered based on (semi) quantitative twohybrid interaction data (binding to CNOT7 and CNOT8); inhibition of cell cycle progression upon expression of BTG1 (percentage of cells in S-phase), and the influence on luciferase expression (Luc mRNA compared to empty vector control) and activity (Luc activity compared to empty vector control). The heat map was generated using the Morpeus tool (https://software.broadinstitute.org/morpheus). 


\section{Supplementary Data}

Supplementary Materials and methods.

Supplementary Figure 1.

Supplementary Figure 2.

Supplementary Figure 3.

Supplementary Table 1. 

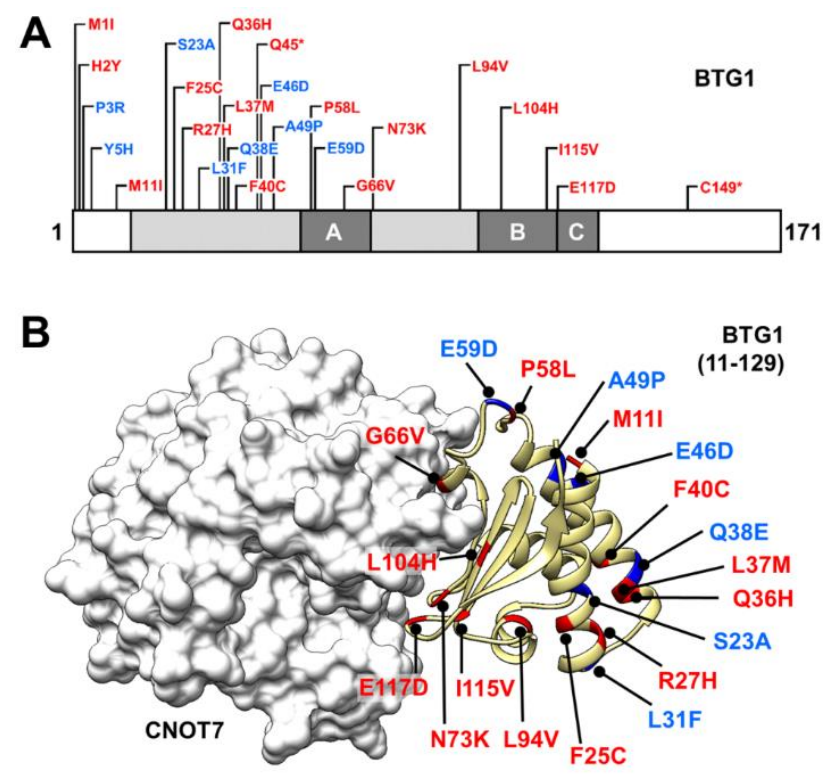

Figure 1. 


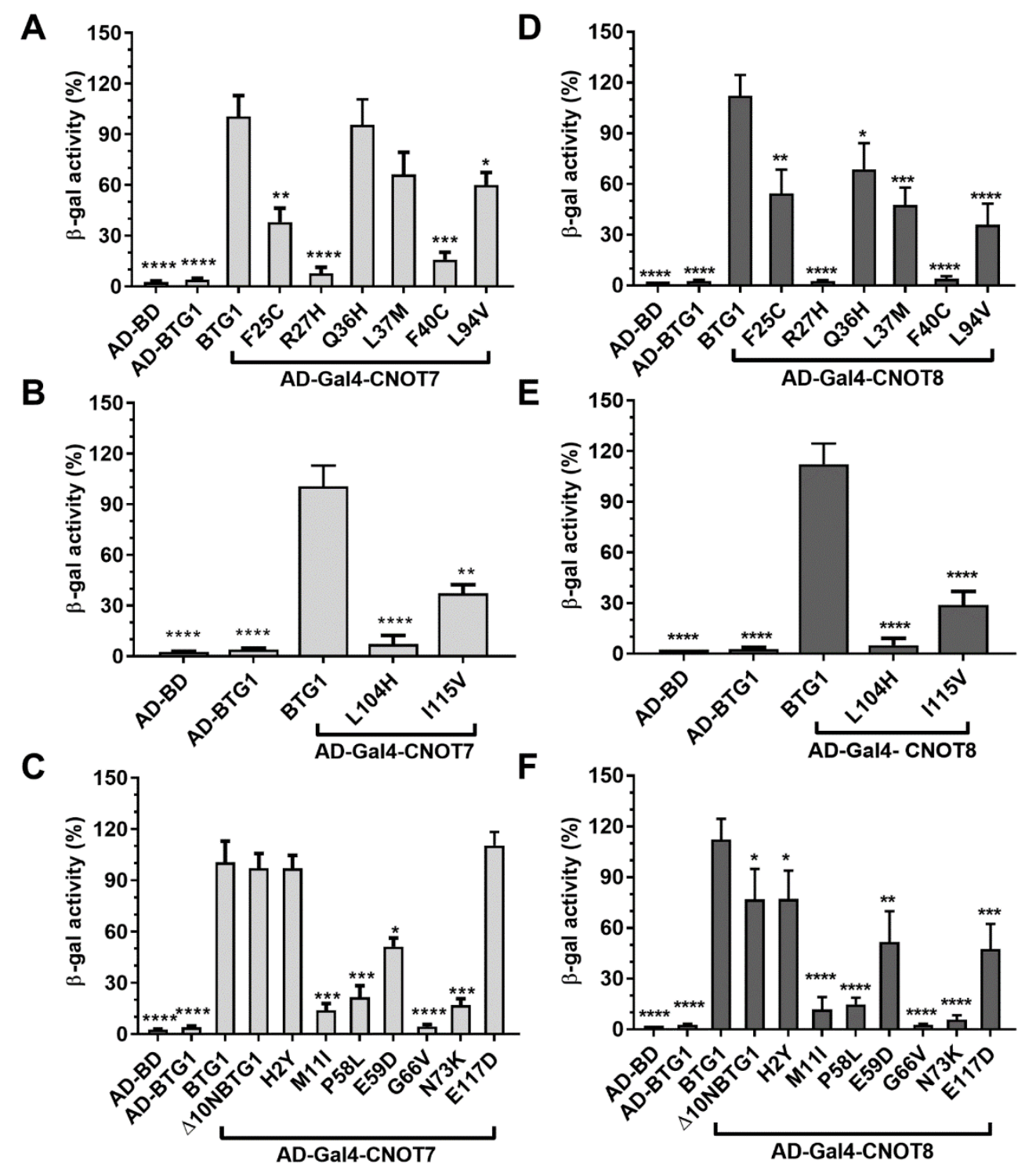

Figure 2. 

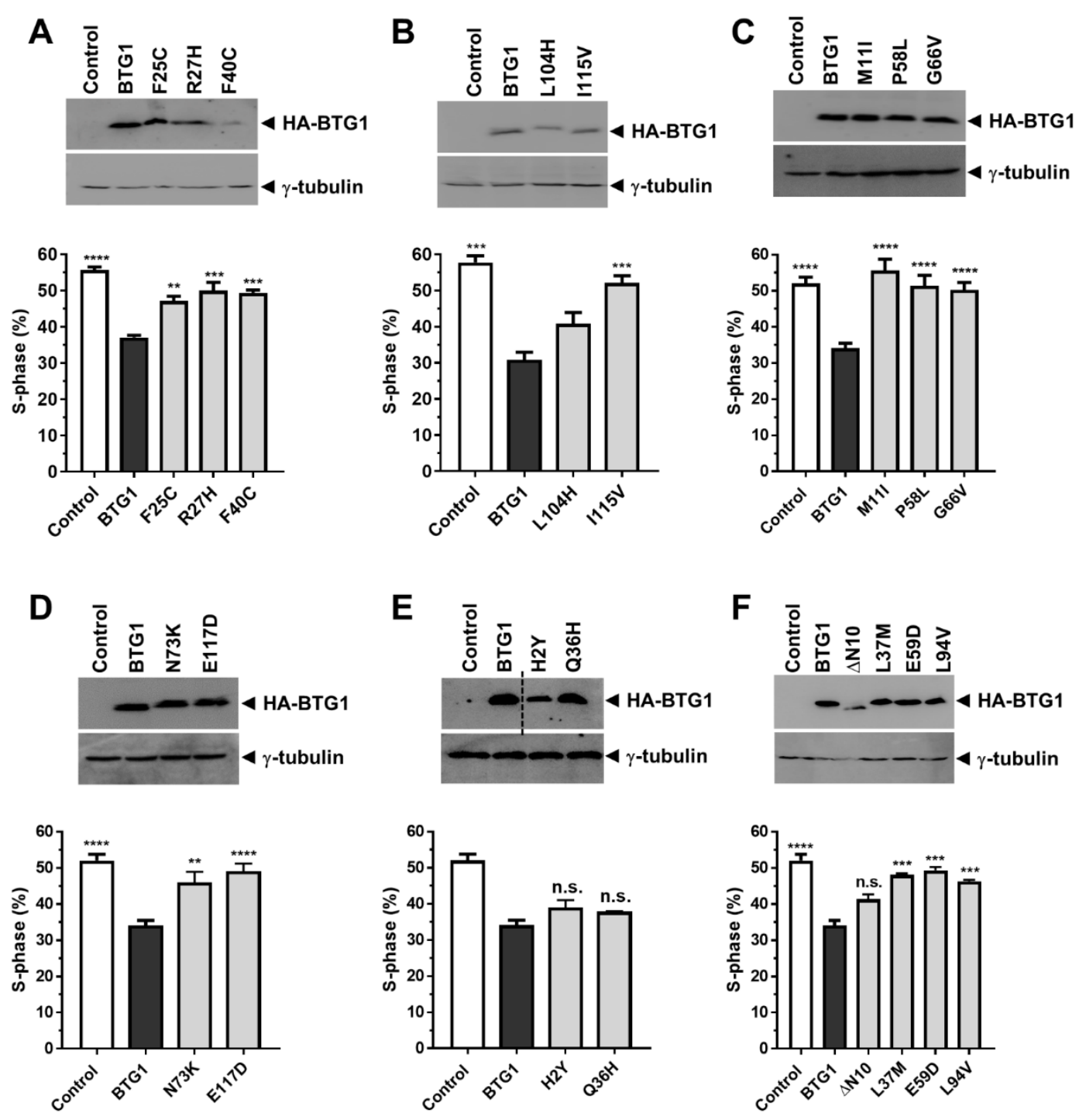

Figure 3. 
A
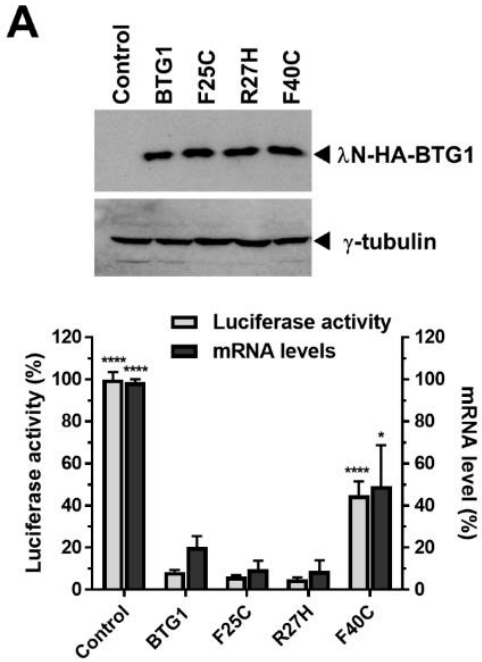

D
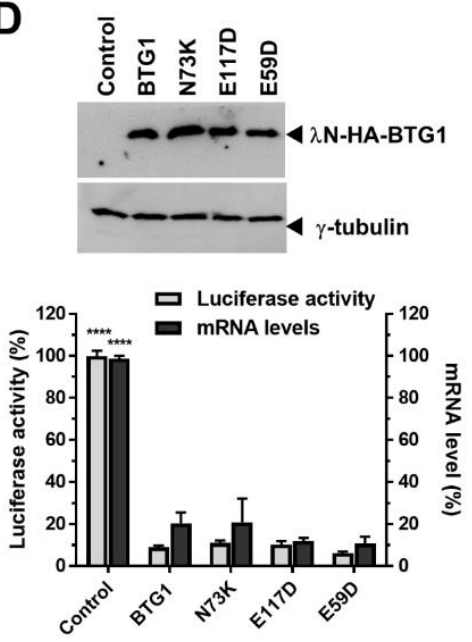

Figure 4.
B
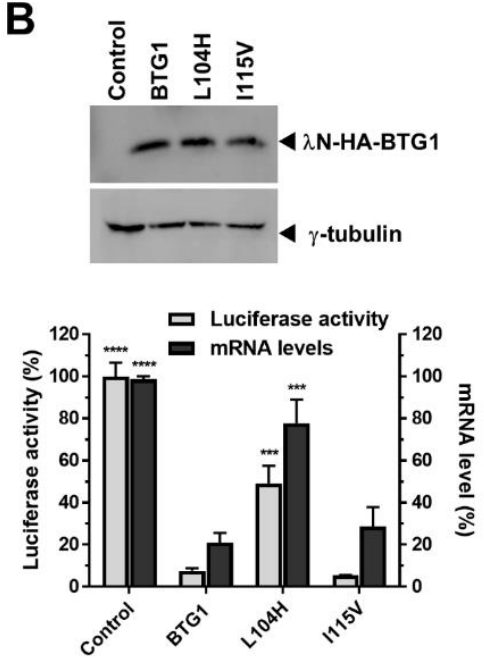

E
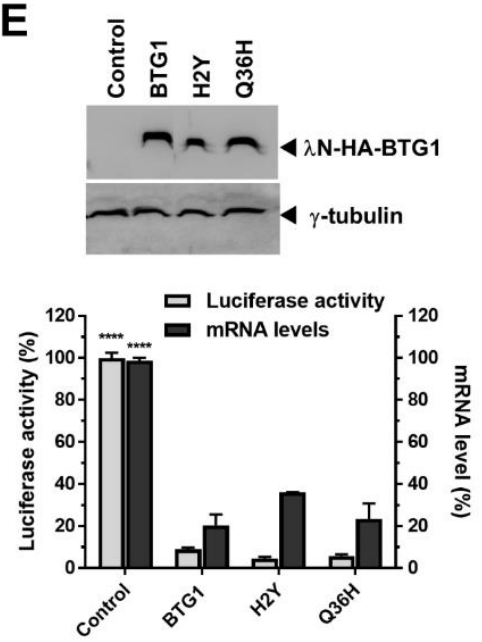

C
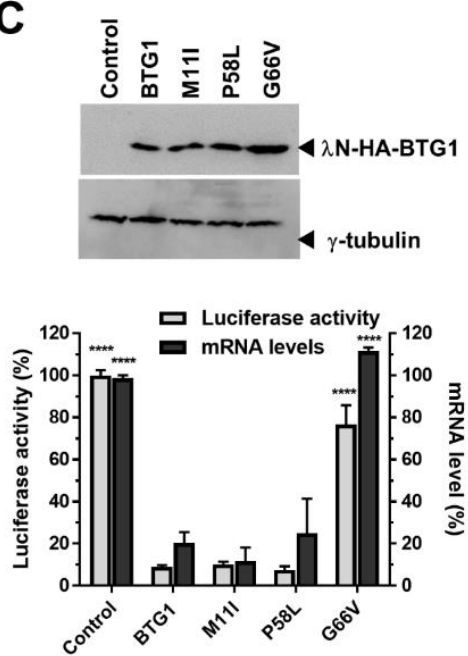

F
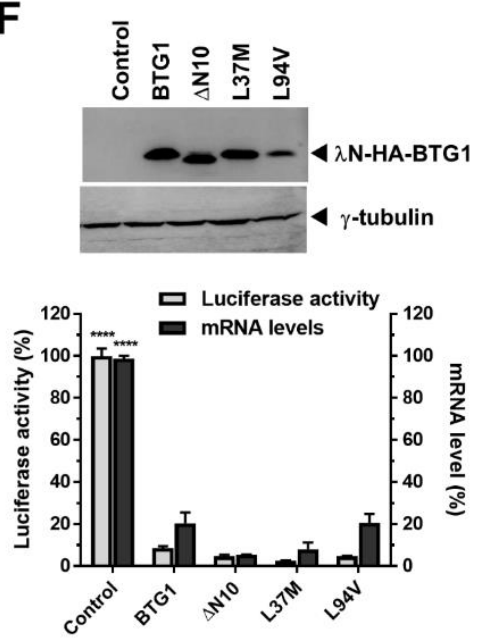


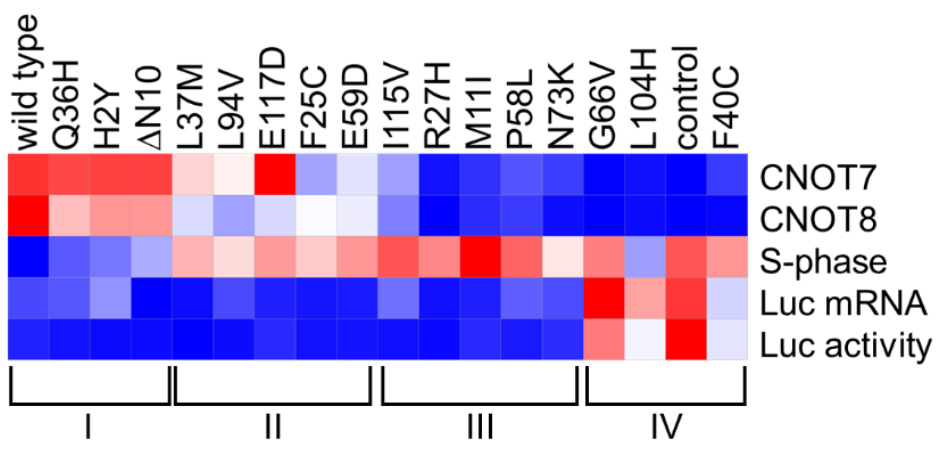

Figure 5. 